\title{
A statistical study on the correlations between plasma sheet and solar wind based on DSP explorations
}

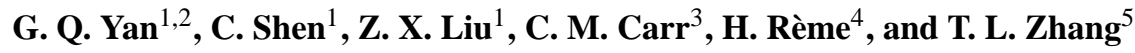 \\ ${ }^{1}$ Key Laboratory of Space Weather, Center for Space Science and Applied Research, Chinese Academy of Sciences, Beijing \\ 100080, China \\ ${ }^{2}$ Graduate School of Chinese Academy of Sciences, No. 19 Jia, Yu Quan Road, Beijing 100039, China \\ ${ }^{3}$ Imperial College of Science, Technology and Medicine, London, UK \\ ${ }^{4}$ CESR, BP4346, 31028 Toulouse Cedex 4, France \\ ${ }^{5}$ Space Research Institute, Austrian Academy of Sciences, Graz, Austria
}

Received: 15 February 2005 - Revised: 6 September 2005 - Accepted: 21 September 2005 - Published: 8 November 2005

Part of Special Issue "Double Star - First Results"

\begin{abstract}
By using the data of two spacecraft, TC-1 and ACE (Advanced Composition Explorer), a statistical study on the correlations between plasma sheet and solar wind has been carried out. The results obtained show that the plasma sheet at geocentric distances of about 9 13.4 Re has an apparent driving relationship with the solar wind. It is found that (1) there is a positive correlation between the duskward component of the interplanetary magnetic field (IMF) and the duskward component of the geomagnetic field in the plasma sheet, with a proportionality constant of about 1.09. It indicates that the duskward component of the IMF can effectively penetrate into the near-Earth plasma sheet, and can be amplified by sunward convection in the corresponding region at geocentric distances of about 9 13.4 Re; (2) the increase in the density or the dynamic pressure of the solar wind will generally lead to the increase in the density of the plasma sheet; (3) the ion thermal pressure in the near-Earth plasma sheet is significantly controlled by the dynamic pressure of solar wind; (4) under the northward IMF condition, the ion temperature and ion thermal pressure in the plasma sheet decrease as the solar wind speed increases. This feature indicates that plasmas in the near-Earth plasma sheet can come from the magnetosheath through the LLBL. Northward IMF is one important condition for the transport of the cold plasmas of the magnetosheath into the plasma sheet through the LLBL, and fast solar wind will enhance such a transport process.
\end{abstract}

Keywords. Magnetospheric Physics (Magnetosheath, Plasma sheet, magnetotail boundary layers)

Correspondence to: G. Q. Yan

(gqyan@ns.spaceweather.ac.cn)

\section{Introduction}

The plasma sheet, where a lot of complicated processes occur, plays an important role in the energy and mass transport from the solar wind into the magnetosphere (Borvosky et al., 1998). The neutral sheet is originally defined as a region where the tail's magnetic field reverses its direction and has a very weak magnitude (Ness, 1965); it has also been defined by some researchers as the midsurface of the nightside plasma sheet (Dandouras, 1988), or as a region within the current sheet where the component in the Sun-Earth direction of the magnetic field is less than the northward component (Shen et al., 2003). Many researchers have simply defined the neutral sheet as a curved surface where the $\mathrm{X}$ component of the geomagnetic field changes its direction, which characterizes the properties of the plasma sheet, from sunward to antisunward and vice versa (Speiser and Ness, 1967; Dandouras, 1988; Xu et al., 1991). However, the neutral sheet is not only a reference surface but also a structure directly connected to the formation of the plasma sheet (Dandouras, 1988). The properties in the plasma sheet are very important for revealing the mechanisms of the transporting of solar wind into the magnetosphere. The purpose of choosing the neutral sheet crossings as data points is to ensure that the spacecraft is located within the plasma sheet and then measures the properties of the plasma sheet. Fairfield (1979), Lui (1986), Tsurutani et al. (1984), Sergeev (1987) and Borovsky et al. (1998) got a linear relationship between the duskward component of the IMF and the duskward component of the geomagnetic field, with proportionality constants of about $0.13,0.13,0.09 \sim 0.21,0.60$ and 0.76 , respectively. Cowley (1981), Moses et al. (1985) and Kaymaz et al. (1994) have discussed in more detail about the transferring of the IMF Y component into the tail region of the magnetosphere. By using a statistical method, Borvosky et al. (1998) have 


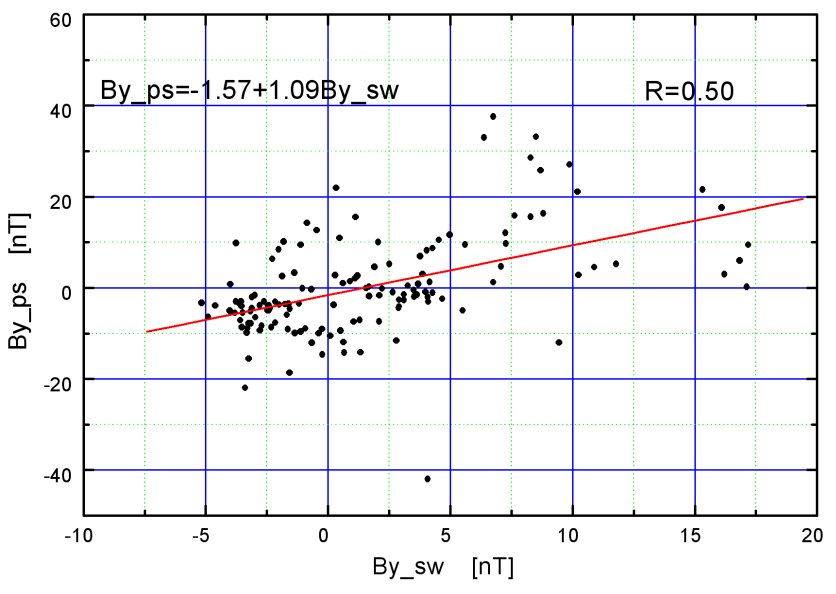

Fig. 1. The relationship between $B_{y}$ in solar wind and that in plasma sheet in GSM coordinates.

investigated the relationship between the plasmas in the tail region plasma sheet with geocentric distances of about $20 \mathrm{Re}$ and that of the solar wind. Their results show the plasma sheet density's strong correlation with the density and dynamic pressure in solar wind, and that the plasma sheet thermal pressure is strongly controlled by solar wind dynamic pressure. However, the properties of the plasma sheet depend on the geocentric distances (Slavin et al., 1985; Nishida, 2000).

In this work, we have investigated the correlations between solar wind and near-Earth plasma sheet. In Sect. 2, the data are described; Sect. 3 presents the results of the investigations; Sect. 4 gives the summary and conclusions.

\section{Data}

The Double Star Programme (DSP) (Liu et al., 2005; Shen and Liu, 2005, this issue) provides a precious opportunity for investigating various regions of the near-Earth magnetosphere. From July through October of 2004, the equatorial spacecraft (TC-1) of DSP could cross the near-Earth plasma sheet of the magnetotail. The observations of TC-1, together with that of the ACE (Advanced Composition Explorer) spacecraft at L1 point, can be used to study the driving relationship between the solar wind and the Earth plasma sheet. The apogee of TC- 1 is $13.4 \mathrm{Re}$, and the ACE spacecraft is located at the L1 point, which is about $235 \mathrm{Re}$ upstream of the Earth in the solar wind. During the four months from July through October of 2004, the orbits of TC-1 have swept the near-Earth magnetotail with geocentric distances less than 13.4 Re. In addition, the spacecraft can cross the neutral sheet during each orbit. The neutral sheet crossings can be identified when the $\mathrm{X}$ component of geomagnetic field in Geocentric Solar Magnetic coordinates (GSM) passes the zero point, and by the data with a time resolution of $4 \mathrm{~s}$, measured by the DSP instrument Flux Gate Magnetometer (FGM) (Carr et al., 2005). The Hot Ion Analyzer (HIA) (Réme et al., 2005) measurements with a time resolution of $\sim 4$ s provide some parameters of the plasma sheet, including the density $(n)$, velocity vector $(\boldsymbol{V})$, ion temperature $(T)$, and so on (Réme et al., 2001).

The time lag between the two measurements in the solar wind and plasma sheet can be described as $\mathrm{X} / \mathrm{V}_{s w}$, where $\mathrm{X}$ is the distance from the L1 point to the Earth's orbit. The speed of the solar wind is varying from about 300 to $1000 \mathrm{~km} / \mathrm{s}$. A large speed corresponds to a small lag and vice versa. In this study, the simultaneous solar wind measurements have been used to match the measurements in the Earth's neutral sheet, providing the properties of solar wind, such as IMF and solar wind speed, density and temperature and so on. Using the data available of TC- 1 and data of ACE, we obtain 139 data points in neutral sheet which are corresponding to the ACE solar wind measurements simultaneously. In addition, all the vectors have been transformed into those in the GSM coordinates. The geocentric distances of the neutral sheet crossings range from 9 to $13.4 \mathrm{Re}$. A Gaussian fitting has been applied in the analysis, because it provides a line approaching the data points by minimizing the errors $\left(\Delta x^{2}+\Delta y^{2}\right)^{1 / 2}$, which is better than a linear fitting. According to the principle of Hypothesis Tests, there exists a threshold for a correlation coefficient described as $R_{\text {random }}=2 / N^{1 / 2}$, in which $\mathrm{N}$ is the sample size, the number of data points. If $|R|>R_{\text {random, there is a correlation at }}$ the confidence degree of $95 \%$; otherwise, there is no correlation (Beyer, 1966; Bendat and Piersol, 1971). In this work, the number of data points is $N=139$, so the threshold is $R_{\text {random }}=0.17$.

In Sect. 3, we will investigate (1) the correlation between the $\mathrm{Y}$ component of IMF in GSM and the magnetic field in the plasma sheet, (2) the density, temperature and ion's thermal pressure in the plasma sheet, influenced by solar wind speed, density and pressure. In Sect. 4, the summary and conclusions will be given.

\section{Results of the correlations between solar wind and the plasma sheet parameters}

The transfer of the IMF Y component into the magnetotail has been talked about by different researchers (Cowley, 1981; Hammond et al., 1992; Fairfield, 1979; Lui 1986; Sergeev, 1987; Tsurutani et al., 1984; Kaymaz et al., 1994). Cowley (1981) interpreted it as the response of the magnetosphere to the torque imposed on the magnetotail by the IMF, while Moses et al. (1985) interpreted it as the plasma sheet field tilted due to convection with different directions in the two hemispheres. As shown in Fig. 1, the $B_{y}$ in the solar wind and that in the plasma sheet have a strong positive correlation, with a coefficient of 0.50 . The proportionality constant here is 1.09 , larger than unity. The proportionality constant is also called the penetration factor by some researchers (Borvosky et al., 1998), which means the ratio of $B_{y}$ in the plasma sheet and $B_{y}$ in the solar wind. As a comparison, Fairfield (1979), Lui (1986), Tsurutani et al. (1984), Sergeev (1987) and Borovsky et 


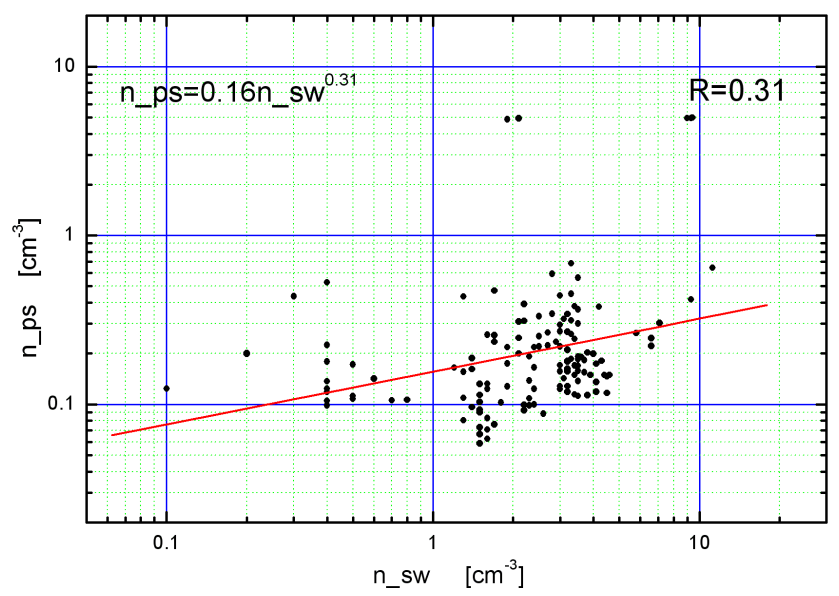

Fig. 2. The relationship between the density of the solar wind and that in the plasma sheet.

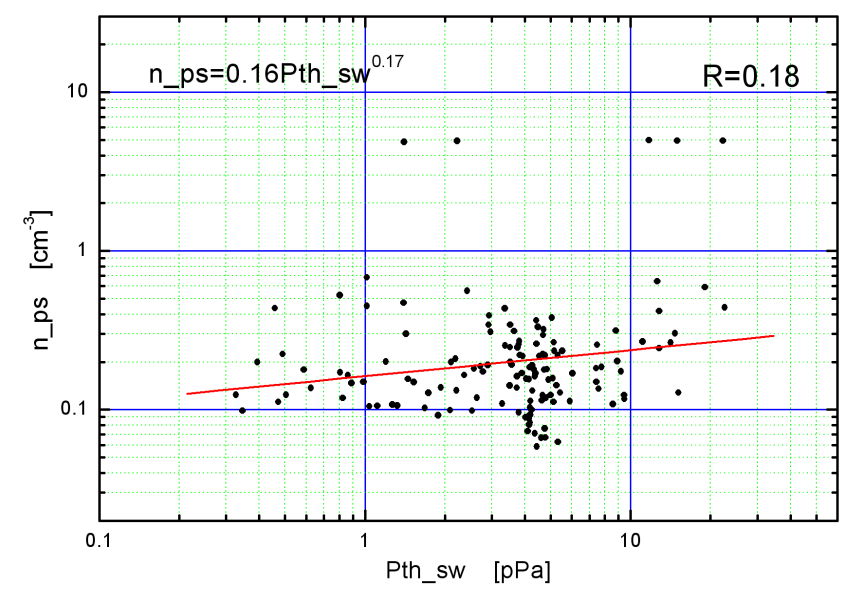

Fig. 3. The relationship between the ion thermal pressure of the solar wind and density in the plasma sheet.

al. (1998) obtained a different penetration factor of 0.13 , $0.13,0.09 \sim 0.21,0.60$, and 0.76, respectively. Kaymaz et al. (1994) compared the previous results on this problem and it can be seen that the proportionality constant or penetration factor increases with the decreasing distance. And our result is consistent with such a trend. Voigt and Hilmer (1987) interpreted the $B_{y}$ in magnetotail as a smaller penetration component and a larger convection-induced component in plasma sheet. Hau and Erickson (1995) also gave a theoretic analysis of how the observed $B_{y}$ in plasma sheet can be amplified when convecting inward on the closed flux tubes. The proportionality constant of 1.09 in this work means that the observed $B_{y}$ in plasma sheet is due to not only the penetration from IMF $B_{y}$, but also due to the amplification by the sunward convection in this region. This may be why the proportionality constant is larger than unity, and penetration result is included in it.

Here we have investigated the relationship between the density of the solar wind and the density in the near-Earth plasma sheet, as demonstrated in Fig. 2. In the tail region

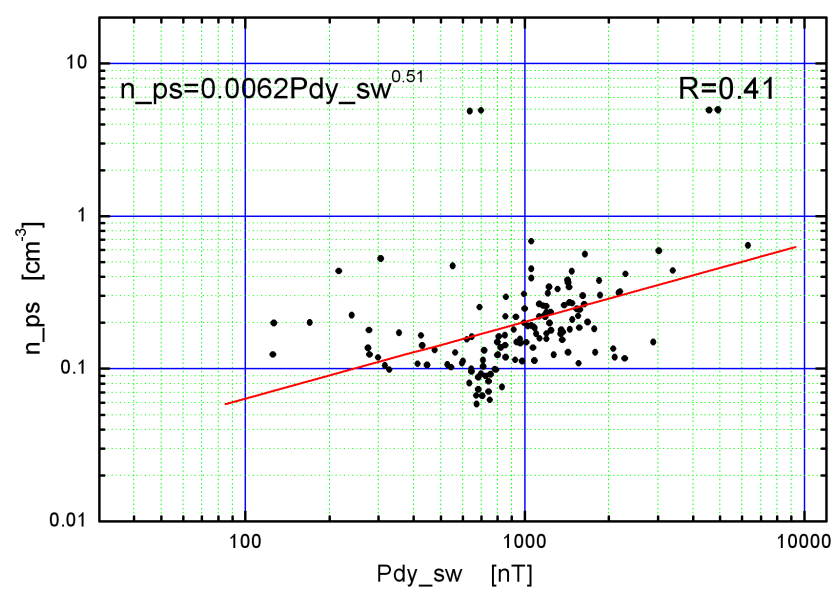

Fig. 4. The relationship between the dynamic pressure of the solar wind and density in the plasma sheet.

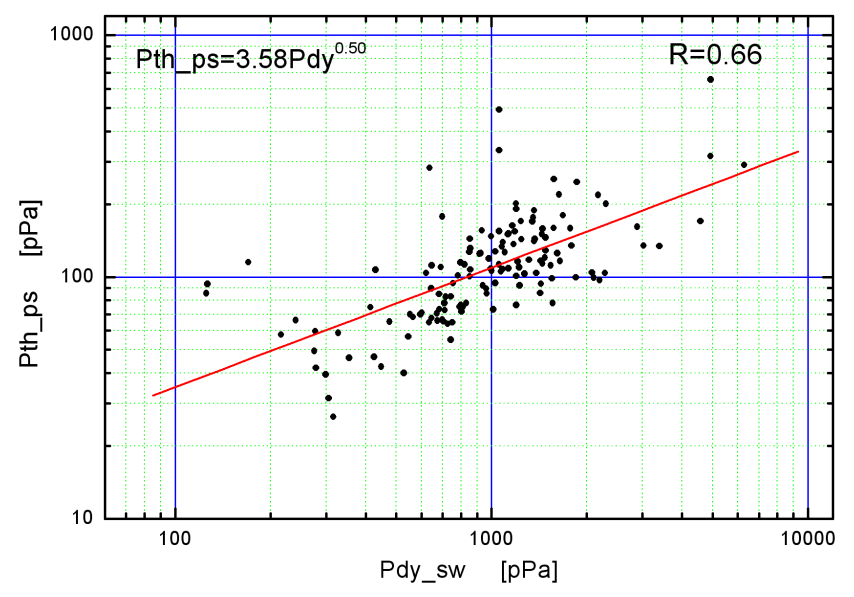

Fig. 5. The relationship between the dynamic pressure of the solar wind and the ion thermal pressure in the plasma sheet.

at about $9 \sim 13.4 \mathrm{Re}$, there is a correlation coefficient of 0.31 . This statistical feature possibly reflects that the plasmas in the plasma sheet may come from the solar wind. However, in the magnetotail region with geocentric distance of about $17.5 \sim 22.5 \mathrm{Re}$, the solar wind density has a rather strong control upon the density in the plasma sheet, with a correlation coefficient of 0.74, as illustrated by Borovsky et al. (1998).

As Figs. 3 and 4 show, the thermal pressure of the solar wind has a weak correlation with the density in the nearEarth plasma sheet, while the correlation between the density in the plasma sheet and the solar wind dynamic pressure is larger, with a value of 0.41 . So we can see that the density in the plasma sheet is influenced by the solar wind dynamic pressure rather than by the solar wind ion thermal pressure. The dynamic pressure of the solar wind plays a more important role during the plasma transport of solar wind into magnetosphere. In fact, the dynamic pressure is much larger than the thermal pressure in the solar wind.

Figure 5 shows that there is a strong, positive correlation between the thermal pressure of ions in the plasma sheet and 


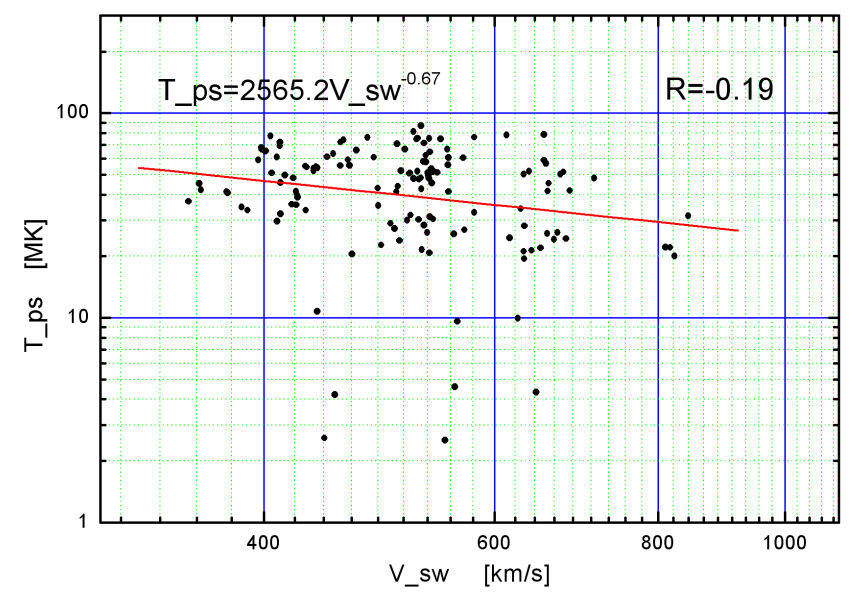

Fig. 6. The relationship between the speed of the solar wind and the ion temperature in the plasma sheet.

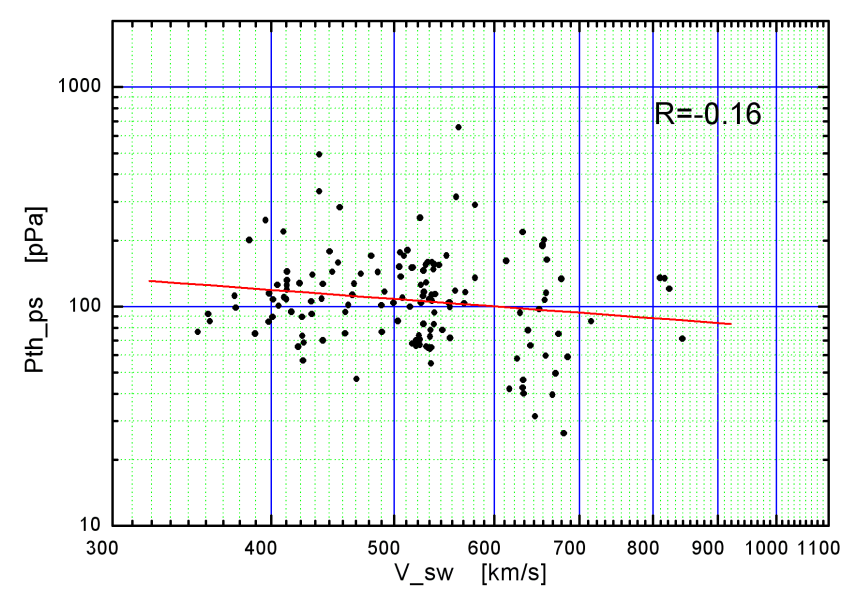

Fig. 7. The relationship between the speed of the solar wind and the ion thermal pressure in the plasma sheet.

the dynamic pressure of the solar wind. Tsyganenko and Mukai (2003) set up analytical models of the central plasma sheet properties as functions of solar wind and IMF parameters. Their model of the plasma sheet thermal pressure indicated that ion thermal pressure in the plasma sheet is controlled by solar wind dynamic pressure. The correlation in Fig. 5 means that in the tail region at $9 \sim 13.4 \mathrm{Re}$, the ion thermal pressure in the plasma sheet is strongly controlled by solar wind dynamic pressure. The ion dynamic pressure dominates in the solar wind, while the ion thermal pressure dominates in the plasma sheet. The strong correlation reflects the pressure balance between the solar wind and the magnetotail plasma sheet.

Figure 6 shows that there is a weak, negative correlation between solar wind speed and ion temperature in the plasma sheet. The correlation coefficient is -0.19 , not very high. On the other hand, there is no correlation between solar wind speed and ion thermal pressure in plasma sheet, as illustrated in Fig. 7.

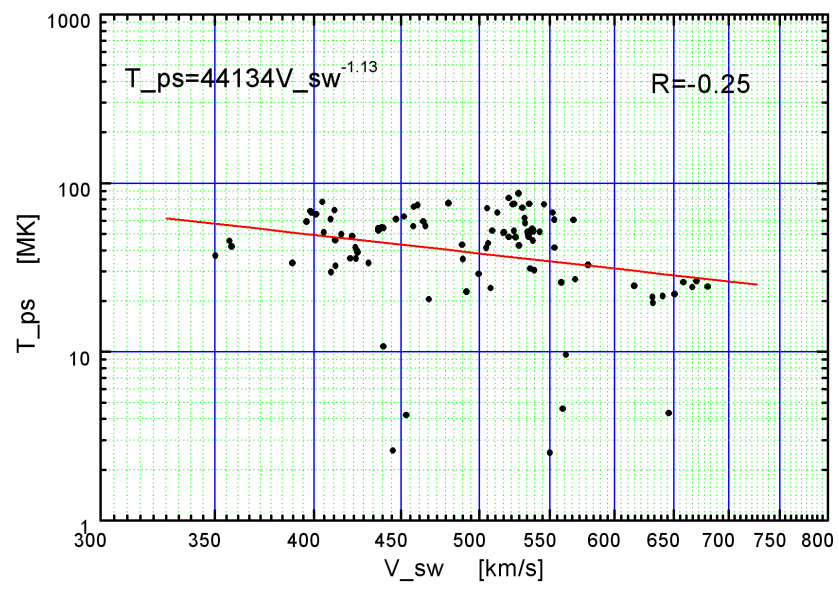

Fig. 8. The relationship between the speed of the solar wind and the ion thermal pressure in the plasma sheet while IMF Bz $>-2.0 \mathrm{nT}$.

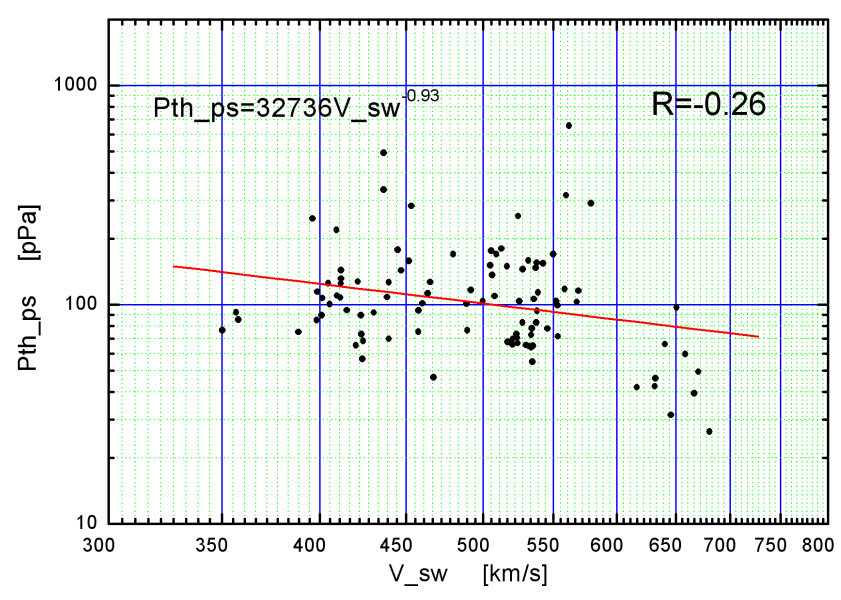

Fig. 9. The relationship between the speed of the solar wind and the ion thermal pressure in the plasma sheet while IMF $\mathrm{B}_{z}>-2.0 \mathrm{nT}$.

Now the separation according to the IMF $B_{z}$ has been made. Data points with IMF $\mathrm{Bz} \leq-2$. $0 \mathrm{nT}$ are regarded as the situation of southward IMF, while the other data points are regarded as the situation of northward IMF. According to such a regulation, the 139 data points are divided into the situation of southward IMF, including $41\left(R_{\text {random }}=0.31\right)$ data points and northward IMF, including $98\left(R_{\text {random }}=0.20\right)$ data points. Under the southward IMF, neither ion temperature nor ion thermal pressure in the plasma sheet has a correlation with the solar wind speed (not shown). However, under northward IMF, both ion temperature and ion thermal pressure have negative correlations with the solar wind speed, with the correlation coefficients of -0.25 and -0.26 , respectively, as shown in Figs. 8 and 9.

The negative correlation in Fig. 8 means that ion temperature in the plasma sheet decreases with the rising of solar wind speed. This feature in the near-Earth plasma sheet is opposite to the positive correlation at the geocentric distance of about $20 \mathrm{Re}$, which has been revealed by Borovsky et al. (1998). Spence and Kivelson (1993) and Fujimoto 
et al. (1996) have pointed out that solar wind plasma enters the magnetotail plasma sheet through the Low Latitude Boundary Layer (LLBL). Terasawa et al. (1997) and Fujimoto et al. (1997) found that plasma sheet becomes colder and denser under northward IMF, and that such things happen mostly near the tail's flanks. From this negative correlation in Fig. 8, it can be implied that in the plasma sheet at geocentric distances of $9 \sim 13.4 \mathrm{Re}$, under northward IMF, ions can be transported to the near-Earth plasma sheet from the LLBL, as well as from the distant tail. The northward IMF is a condition for such transportation through the LLBL. Ions from the LLBL are relatively cold, while ions from the distant tail are relatively hot (Nishida, 2000; Hasegawa et al., 2004a). When the solar wind speed is higher, more ions with low temperature enter the near-Earth plasma sheet through the LLBL, thus the temperature of the plasma sheet becomes colder. That is to say, under northward IMF, besides the distant tail sources, LLBL also plays an important role in the plasma transport into the near-Earth plasma sheet at the geocentric distances of 9 13.4 Re. The ion thermal pressure in the plasma sheet decreases with the increase in the solar wind speed, shown in Fig. 9, further supporting the viewpoint that, under northward IMF, faster solar wind will enhance the transfer of the cold plasmas in the magnetosheath into the magnetosphere through the LLBL. Comparing the results here with the results of Borovsky et al. (1998), it could also be inferred that the LLBL transferring process more likely occurs at the near-Earth LLBL with geocentric distances less than $20 \mathrm{Re}$. This statistical result also supports the point that, the faster the solar wind, the more effective the transfer of the plasmas from the magnetosheath into magnetosphere through LLBL (Hasegawa et al., 2004b).

\section{Summary and conclusions}

In this study, the driving relationship of the near-Earth plasma sheet by the solar wind has been investigated by using a statistical method. The solar wind data were time shifted to correct for solar wind propagation. The results and conclusions obtained may be summarized as follows.

The $B_{y}$ in the solar wind and the $B_{y}$ in the plasma sheet have a strong, positive correlation, with a coefficient of 0.50 . The proportionality constant is as large as 1.09 ; it is possibly due to both the penetration of the IMF $B_{y}$ and the amplification by the sunward convection in the concerned region. This result would support the model of Hau and Erickson (1995).

The density in the near-Earth plasma sheet can be influenced by the solar wind density and the solar wind dynamic pressure. The dynamic pressure of the solar wind plays an important role during the plasma transport of the solar wind into the magnetosphere, while the thermal pressure of the solar wind has less influence on the density of the near-Earth plasma sheet.

The analytical model of Tsyganenko and Mukai (2003) for the plasma sheet thermal pressure indicated that ion thermal pressure in the plasma sheet is controlled by solar wind dy- namic pressure. There is a similar result in our work. The strong, positive correlation between the thermal pressure of ions in the plasma sheet and the solar wind dynamical pressure reflects the requirement of a pressure balance in a statistical sense.

Under northward IMF, the ion temperature and ion thermal pressure decrease with the increase in the solar wind speed. Such negative correlations (although weak) may imply that a fast solar wind will enhance the transfer of the cold plasmas in the magnetosheath through the LLBL, and northward IMF is possibly one important condition for such transportation. The result is consistent with the results of some previous researchers (Tsyganenko and Mukai, 2003).

Acknowledgements. The authors are grateful to ACE swepam \& mag team for offering the ACE measurement data. The authors are also grateful to DSP FGM \& HIA team for offering DSP magnetic and hot ion measurement data. This work was supported by National Natural Science Foundation of China Grant No. 40125012 and 40390150, and Ministry of Science and Technology of China Grant No. G20000784.

Topical Editor T. Pulkkinen thanks V. A. Sergeev, G. Siscoe and another referee for their help in evaluating this paper.

\section{References}

Bendat, J. S. and Piersol, A. G.: Random Data: Analysis and Measurement Procedures, Sect. 4.8.1, John Wiley, New York, 1971.

Beyer, W. H. (Ed.): Handbook of Tables for Probability and Statistics, Sect. IX, Chem. Rubber, Cleveland, Ohio, 1966.

Borvosky, J. E., Thomsen, M. F., and Elphic, R. C.: The driving of plasma sheet by the solar wind, J. Geophys. Res., 103, $17617-$ $17639,1998$.

Carr, C., Brown, P., Zhang, T. L., et al.: The Double Star magnetic field investigation: instrument design, performance and highlights of the first year's observation, Ann. Geophys., 23, 27132732, 2005.

Cowley, S. W. H.: Magnetospheric asymmetries associated with the Y-component of the IMF, Planet. Space. Sci., 29, 79-76, 1981.

Dandouras, J.: On the average shape and position of the geomagnetic neutral sheet and its influence on plasma sheet statistical studies, J. Geophys. Res., 97, 7345, 1988.

Fairfield, D. H.: On the average configuration of the geomagnetic tail, J. Geophys. Res., 84, 1950, 1979.

Fujimoto, M., Nishida, A., Mukai, T., Saito, Y., Yamamoto, T., and Kokubun, S.: Plasma entry from the flanks of the near-Earth magnetotail: GEOTAIL observations in the dawnside LLBL and the plasma sheet, J. Geomag. Geoelectr., 48, 711-727, 1996.

Fujimoto, M.; T. Terasawa, and T. Mukai : The cold-dense plasma sheet: A GEOTAIL perspective, Space Science Reviews, 80, 325-339, 1997.

Hammond, C. M., Kivelson, M. G., and Walker, R. J.: Planetary magnetotails: magnetic configuration and nonthermal plasma, Ph.D. thesis, Univ. of Calif., LA, 1992.

Hasegawa, H., Fujimoto, M., Saito, Y., and Mukai, T.: Dense and stagnant ions in the low-latitude-boundary-region under northward interplanetary magnetic field, Geophys. Res. Lett., 31, L0682, doi:10.1029/2003GL019120, 2004a. 
Hasegawa, H., Fujimoto, M., Phan, T. D., et al.: Transport of solar wind into magnetosphere through rolled-up Kelvin-Helmholtz vortices, Letters to Nature, 430, 755-758, 2004b.

Hau, L.-N. and Erickson, G. M.: Penetration of the interplanetary magnetic field $B_{y}$ into Earth's plasma sheet, J. Geophys. Res., 100, 21 745-21 751, 1995.

Kaymaz, Z., Siscoe, G. L., and Luhmann, J. G.: Interplanetary magnetic field control of magnetotail magnetic field geometry: IMP 8 observations, J. Geophys. Res., 99, 11 113-11 126, 1994.

Liu, Z. X., Escoubet, C. P., Pu, Z., Laakso, H., Shi, J. K., Shen, C., and Hapgood, M.: The Double Star mission, Ann. Geophys., 23, 2707-2712, 2005.

Lui, A. T. L.: Solar wind influence on configuration and dynamics, in Solar Wind-Magnetosphere coupling, edited by: Kamide, Y. and Slavin, J. A., Kluwer, Norwell, Mass., p. 671, 1986.

Moses, J. J., Crooker, N. U., Gorney, D. J., Siscoe, and G. L.: High latitude convection on open and closed field lines for large IMF $B_{y}$, J. Geophys. Res., 90, $11078,1985$.

Ness, N. F.: The earth's magnetotail, J. Geophys. Res., 70, 29893005, 1965.

Nishida, A.: The Earth's Dynamic Magnetotail, Space Sci. Rev., 91, 507-577, 2000.

Rème, H., Aoustin, C., Bosqued, J. M., et al.: First multispacecraft ion measurement in and near the Earth's magnetosphere with the identical Cluster ion spectrometry (CIS) experiment, Ann. Geophys., 19, 1303-1354, 2001,

SRef-ID: 1432-0576/ag/2001-19-1303.

Rème, H., Dandouras, I., Aoustin, C., et al.: The HIA instrument on board the Tan Ce 1 Double Star near-equatorial spacecraft and its first results, Ann. Geophys., 23, 2757-2774, 2005.

Sergeev, V. A.: Penetration of the $B_{y}$-component of the interplanetary magnetic field (IMF) into the Tail of the Magnetosphere, Geomagn. Aerono., 27, 4, 1987.
Shen, C., Li, X., Dunlop, M., Liu, Z. X., et al.: Analyses on the geometrical structure of magnetic field in the current sheet based on cluster measurements, J. Geophys. Res., 108, 1168-1182, 2003.

Shen, C. and Liu, Z. X.: Double Star project - master science operations plan, Ann. Geophys., 23, 2851-2859, 2005.

Slavin, J. A., Smith, E. J., Sibeck, D. G., Baker, D. N, Zwichkl, R. D., and Akasolf, S.-I.: An ISEE-3 Study of Average and Substorm Conditions in the Distant Magnetotail, J. Geophys. Res., 90, 10 875-10895, 1985.

Speiser, T. W. and Ness, N. F.: The neutral sheet in the geomagnetic tail: Its motion equivalent currents, and field line connection through it, J. Geophys. Res., 72, 131, 1967.

Spence, H. E. and Kivelson, M. G.: Contributions of the lowlatitude boundary layer to the finite widh magnetotail convection model, J. Geophys. Res., 98, 15 847, 1993.

Terasawa, T., Fujimoto, M., Mukai, T., Shinohara, I., Saito, Y., Yamamoto, T., Machida, S., Kokubun, S., Lazarus, A. J., Steinberg, J. T., and Lepping, R. P.: Solar wind control of density and temperature in the near-Earth plasma sheet: WIND-GEOTAIL collaboration, Geophys. Res. Lett., 24, 935-938, 1997.

Tsurutani, B. T., Jones, D. E., Lepping, R. P., Smith, E. J., and Sibeck, D. G.: The relationship between the IMF $B_{y}$ and distant tail $\left(150-238 \mathrm{R}_{E}\right)$ lobe and plasma sheet $B_{y}$ fields, Geophys. Res. Lett., 11, 1082, 1984.

Tsyganenko, N. A. and Mukai, T.: Tail plasma sheet models derived from Geotail particle data, J. Geophys. Res., 108(A3), 1136, doi:10.1029/2002JA009707, 2003.

Voigt, G.-H. and Hilmer, R. V.: The influence of the IMF By component on the Earth's magneto-hydrostatic magnetotail, in: Magnetotail Physics, edited by: Lui, A. T., John Hopkins University, Baltimore, Maryland, p. 91, 1987.

$\mathrm{Xu}, \mathrm{R}$. L.: Displayed neutral sheet model observed by the ISEE-2 satellite, J. Atmos. Terrestrial Physics, 53, 12, 1991. 